\title{
Schulleitung zwischen Alltagsbewältigung und Schulentwicklung - Thesen aus einer qualitativen multiperspektivischen Arbeitsplatzanalyse
}

\author{
Christoph Kruse, Westfälische Wilhelms-Universität Münster \\ Stephan Gerhard Huber, Pädagogische Hochschule Zug
}

\begin{abstract}
Der Beitrag geht anhand einer Interviewstudie den Fragen nach, wie Aufgaben und Schnittstellen im Schulmanagement ausgestaltet sind und wie funktional dies geschieht. Ergebnisse von 18 leitfadengestützten Experteninterviews mit Schulleitungen sowie Sekretariatsmitarbeitenden und Schulpflegemitgliedern werden präsentiert und forschungsbezogen diskutiert. Es zeigt sich, dass eine hohe Belastungsintensität sowie permanente Zeitknappheit aus Sicht der Akteure zwangsläufig zu einer Priorisierung der Sicherstellung des Schulalltags führen, wodurch schulqualitätsrelevante Aufgaben systematisch vernachlässigt werden. Angesichts von Unklarheiten und Optimierungspotenzial bezüglich der Arbeitsteilung wird argumentiert, dass Schnittstellen einen entscheidenden Einflussfaktor für das Handeln im Schulmanagement darstellen.
\end{abstract}

\section{Einleitung}

Spätestens seit dem «mässigen Abschneiden der Schweiz» (Ramseier \& Brühwiler, 2003, S. 23) bzw. dem «Pisa-Schock» in Deutschland im Jahre 2001 und der gesteigerten Eigenverantwortung der Einzelschule ist eine verstärkte Bedeutungszuschreibung zur Schulleitungsposition zu beobachten, welche mit einer Kompetenzverschiebung von hierarchisch übergeordneten Instanzen hin zur Schulleitung einhergeht (Bonsen, 2016). In der Schweiz ist für diesen Relevanzgewinn zunächst die Einführung der Schulleitungsposition im Kontext bildungspolitischer Reformen zur sogenannten «geleiteten Schule» konstitutiv. Der Zeitpunkt dieser Einführung (zwischen 1980 und 2010) variiert stark zwischen den Kantonen (vgl. Huber, 2011a, S. 475), im hier untersuchten Feld, dem Kanton Aargau, wurde die Schulleitung zum Jahr 2006 flächendeckend eingeführt ${ }^{1}$. Daher kann im Kontrast zum traditionellen Steuerungsmodell schulischer Bildung (in Deutschland: bürokratische Steuerung, in der Schweiz: vor der Einführung geleiteter Schulen) von grundsätzlich veränderten Aufgaben und Schnittstellentätigkeiten im derzeitigen Schulmanagement der Einzelschule ausgegangen werden.

Das Forschungsvorhaben hat das Ziel, die subjektiven Perspektiven von Schulleitungen, Schulverwaltungen und Schulpflegen als zentrale Akteure im Schulmanagement auf ebendiese Aufgaben und Schnittstellen mittels leitfadengestützter Interviews herauszuarbeiten und theoriegeleitet zu diskutieren. Hierzu wird das Konzept der Arbeitsplatzanalyse genutzt, das anhand offizieller Aufgabenbeschreibungen (2.1) sowie einer Arbeitsanalyse auf Basis theoretischer Überlegungen zum Schulmanagement und zur Belastung (2.2) und einer Rollenanalyse vor dem Hintergrund der Educational Governance (2.3) die Wahrnehmungen der Akteure fokussiert. Ausgehend von bisherigen Forschungsbefunden (3) werden diese Perspektiven im Forschungsdesign bzgl. der Zielsetzung (4) mit einer Qualitativen Inhaltsanalyse ausgewertet. Die Ergebnisse werden gemäss der theoretischen Struktur präsentiert, interpretiert und unter der Berücksichtigung des Forschungsstands zu Thesen verdichtet (5).

\section{Theoretischer Rahmen: Arbeitsplatzanalyse}

In dieser Studie wird das Konzept der Arbeitsplatzanalyse (oft synonym: Arbeitsanalysen, vgl. Greif, 2021) aus der Arbeits- und Organisationspsychologie als theoretischer Rahmen genutzt. Hierzu schliessen wir uns der folgenden Definition an: «Gegenstand der [...] Arbeitsanalyse ist die Analyse und Bewertung von Arbeitstä-

1 Aber auch an aktuellen Reformen lässt sich eine verstärkte Relevanz der Schulleitung bzw. die o. g. Kompetenzverschiebung von übergeordneten Instanzen ablesen: So entschieden die Stimmbürger/-innen des Kantons Aargau am 27.09.2020, dass die Schulpflege per 1. Januar 2022 abgeschafft wird. 
tigkeiten und ihrer Bedingungen sowie die Wirkungen der Arbeitsbedingungen und Anforderungen auf das Individuum» (Schaper, 2019, S. 386). Unter den möglichen Analysekomponenten werden prominent "Arbeitsaufgaben» (ebd.) und «[a]ufgabenbezogene Verhaltensweisen/Anforderungen» (ebd.) gefasst, unter den Zielen die Identifikation von "Gestaltungs- und Optimierungsbedarfe[n] [...] der Arbeitsausführung und -organisation [sowie] gesundheitliche[n] Belastungen [...]» (ebd.). Diesen Zielsetzungen entsprechend wird zum einen der Analysefokus auf die subjektiven Wahrnehmungen der Akteure gelegt (vgl. 4). Zum anderen gilt es, Arbeitsplatzanalysen je nach Fragestellung und Forschungsgegenstand theoretisch zu fundieren. Eine Integration unterschiedlicher Theoriemodelle ist nach Schaper (2019) möglich und geboten, die nun anhand der heuristischen Differenzierung der Arbeitsplatzanalyse nach Aufgaben-, Arbeits- und Rollenanalyse nach Berthel und Becker (2013, vgl. Abb. 1) vorgestellt wird.

\section{Abbildung 1}

Arbeitsplatzanalyse und Analysefokus (eigene Darstellung nach Berthel und Becker, 2013, S. 249)

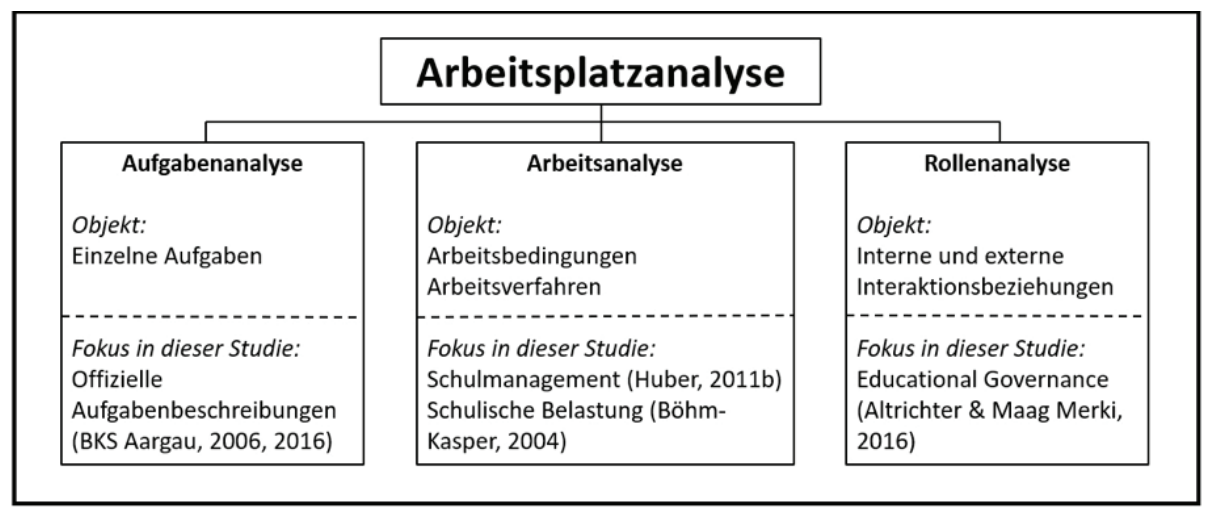

2.1 Aufgabenanalyse: Schulleitungen, Schulpflegen und Schulverwaltungen

Die Aufgabenanalyse bildet nach Berthel und Becker (2013) den «Ausgangspunkt einer Analyse der Arbeitssituation» (S. 248). Hier geht es um systembedingte Rollen- und Aufgabenzuschreibungen, die als Handlungsgrundlage für die hier beforschten Akteursgruppen fungieren. Zum Erhebungszeitpunkt lauten diese wie folgt:

Die Schulpflege ist vom Volk gewählt und ihr obliegt die strategische und politische Führung der Schule, wohingegen der Aufgabenbereich der Schulleitungen die sogenannte operative Führung umfasst (BKS Aargau, 2016).

Die zentralen Aufgaben der Schulpflege werden wie folgt benannt:

- Sie hat die Verantwortung für die Weiterentwicklung der ganzen Schule, für die Einhaltung der kantonalen Vorgaben, die Festlegung der Ziele und lokalen Rahmenbedingungen und die strategische Führung.

- Als Anstellungsbehörde stellt sie Schulleitung und Lehrpersonen an und löst Arbeitsverhältnisse auf.

- Sie führt die Schulleitung und beurteilt sie.

- Sie trifft die beschwerdefähigen Entscheide. Es handelt sich dabei hauptsächlich um Laufbahnentscheide [...] (ebd.)

Die Aufgabengebiete der Schulleitung werden in den folgenden Kategorien zusammengefasst:

- Gestaltung und Entwicklung der Schule (pädagogische Führung)

- Qualitätsentwicklung und Qualitätssicherung

- Personalführung

- Organisation und Administration

- Information und Kommunikation (ebd.)

Für das Schulsekretariat (synonym: Schulverwaltung) wird angeführt:

- Entlastung der Schulleitung und der Schulpflege von administrativen und organisatorischen Aufgaben

- Anlaufstelle für Lehrpersonen, Schülerinnen und Schüler, Eltern, Behörden, Bevölkerung in administrativen Fragen (BKS Aargau, 2006, S. 3)

Somit können die drei Akteursgruppen in eine formale Hierarchie mit der Schulpflege als übergeordnetem, der Schulleitung als mittlerem und der Schulverwaltung als nachgeordnetem Akteur im Schulmanagement eingeordnet werden. Diese Akteursgruppen führen die Volksschule, ein Konglomerat verschiedener Schulstufen (Kindergarten, Primarschule, Realschule, Sekundarschule und ggf. Bezirksschule), die wiederum in unter- 
schiedlichen oder gemeinsamen Schulhäusern untergebracht sind. Je Gemeinde im Kanton Aargau existiert für gewöhnlich eine Volksschule, deren schulstufenbezogene Zusammensetzung und Grösse variiert. In der Folge kann jede der obengenannten Akteursgruppen auch aus mehreren Personen je Volksschule bestehen. Ferner ist aus formaler Sicht offen, inwiefern sich diese Personen die (Leitungs-)Aufgaben innerhalb der Akteursgruppe (z.B. innerhalb der Schulleitung) aufteilen (vgl. Kanton Aargau, 2005). Das zur Verfügung stehende Gesamtpensum für die Schulleitungsfunktion wird nach kantonalem Schlüssel auf Grundlage der Schüler/-innenzahl der jeweiligen Volksschule festgelegt.

\subsection{Arbeitsanalyse: Tätigkeiten und Belastung im Schulmanagement}

Zentral in der Heuristik der Arbeitsplatzanalyse nach Berthel und Becker (2013) ist die Arbeitsanalyse. Hierunter sind die «Gliederung der Erfüllungsvorgänge (Arbeitsverfahren), die Analyse der Arbeitsbedingungen und die Analyse der Mittel zur Aufgabenerledigung» (S. 249, vgl. Abb. 1) bzw. die Analyse der «aufgabenbezogene[n] Verhaltensweisen/Anforderungen» (Schaper, 2019, S. 386) zu fassen. Ausgehend von der Aufgabenanalyse (vgl. 2.1) gilt es zu erforschen, welchen Aufgaben und Tätigkeiten die Akteure in ihrer Praxis tatsächlich nachgehen und wie sich welche aufgabenbezogenen Bedingungen dabei auswirken. Insofern ergibt sich auch hier der Analysefokus auf die subjektiven Wahrnehmungen der Akteure. Als zentrale Komponente der Arbeitsplatzanalyse verlangt dies in besonderer Weise nach theoretischer Fundierung:

Zunächst erscheinen Theorien und Konzepte zur Schulentwicklung naheliegend, deren Kern sich beschreiben lässt «als dauerhafte, bewusste und absichtsvolle sowie systematisierte Weiterentwicklung (im Sinne einer Verbesserung) von Einzelschulen» (Dedering, 2012, S. 46). Es kann hier allerdings nicht vorab angenommen werden, dass es sich bei den analysierten Arbeiten per se um schulentwicklungsbezogene Tätigkeiten handelt. Ebenfalls denkbar wäre eine Bezugnahme auf Konzepte des Educational Leadership (vgl. z. B. die Übersicht bei Tulowitzki \& Pietsch, 2020), welche den Schwerpunkt stärker auf die (potenziell lernförderlichen) Interaktionen von Schulleitungen (insb. mit Lehrpersonen und Schüler/innen) legen. Auch hier erscheint die normative Fokussierung der Tätigkeiten für die Ziele der Arbeitsplatzanalyse ungeeignet. Erforderlich ist somit vielmehr ein Konzept, das (1) den Gegenstand der Leitung einer Einzelschule analytisch zu strukturieren vermag und (2) auf die genannten drei Akteursgruppen gleichermassen anwendbar ist, ohne (3) das hier verfolgte Forschungsinteresse normativ zu stark zu präfigurieren. Zu diesem Zweck nutzen wir konzeptionell das Modell des Schulmanagements, das definiert wird als

professionelles, also qualifiziertes, systematisches und zielgerichtetes Management der Arbeit von Schule sowie der Sicherung und Entwicklung der Qualität dieser Arbeit. [...] Im Rahmen von Schulmanagement wird geklärt, welche Personen mit welchen Aufgaben in welcher Form und in welcher Struktur mit bestimmten Instrumenten (und entsprechendem Verhalten) bestimmte Prozesse ausführen (Huber, 2011b, S. 2).

\section{Abbildung 2}

Komponenten von Schulmanagement (Huber, 2011b)

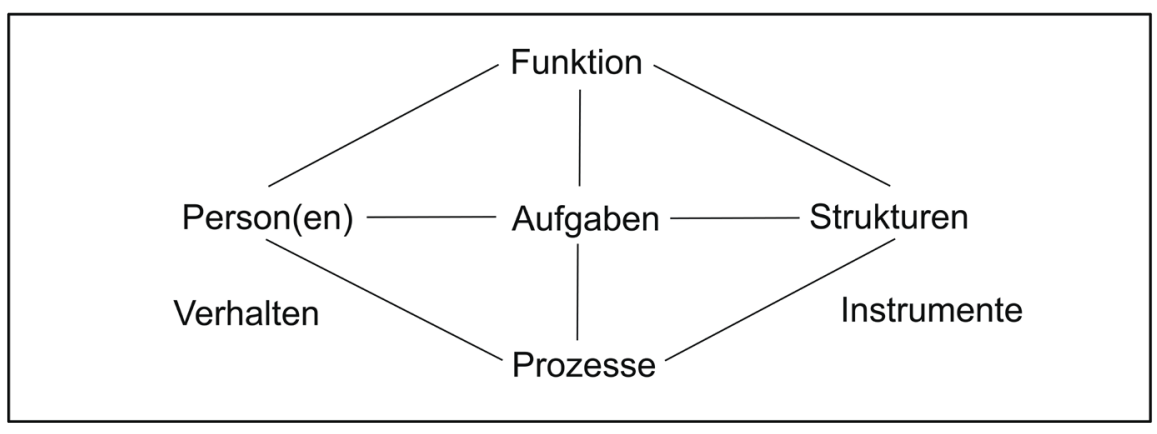

Der Fokus der Studie liegt auf Funktionen, Strukturen und Aufgaben von verschiedenen Personen im Schulmanagement (vgl. Abb. 2). Die Aufgaben werden ferner nach Handlungsfeldern ausdifferenziert, welchen 
wiederum Beispieltätigkeiten zugeordnet werden können (Huber, 2011b; Huber et al., 2014, vgl. Abb. 3)2 Diese Systematik kann zur analytischen Differenzierung des Handelns im Schulmanagement herangezogen werden. Die beiden Modelle (Abb. 2 und 3) werden ferner zur theoretischen Strukturierung von Forschungsstand, Fragestellungen sowie Analyseergebnissen angeführt. So können die von den Akteuren benannten Tätigkeiten in der Arbeitsplatzanalyse strukturiert werden, ohne vorab programmatisch gefasst zu werden.

Dem Nachteil dieses Konzepts, der Vernachlässigung sozialer Interaktionen, soll mit der Ergänzung um die Governance-Perspektive und dessen Betonung der Handlungskoordination (vgl. 2.3) begegnet werden.

\section{Abbildung 3}

Handlungsfelder von Schulmanagement (Huber, 2011b; Huber et al., 2014)

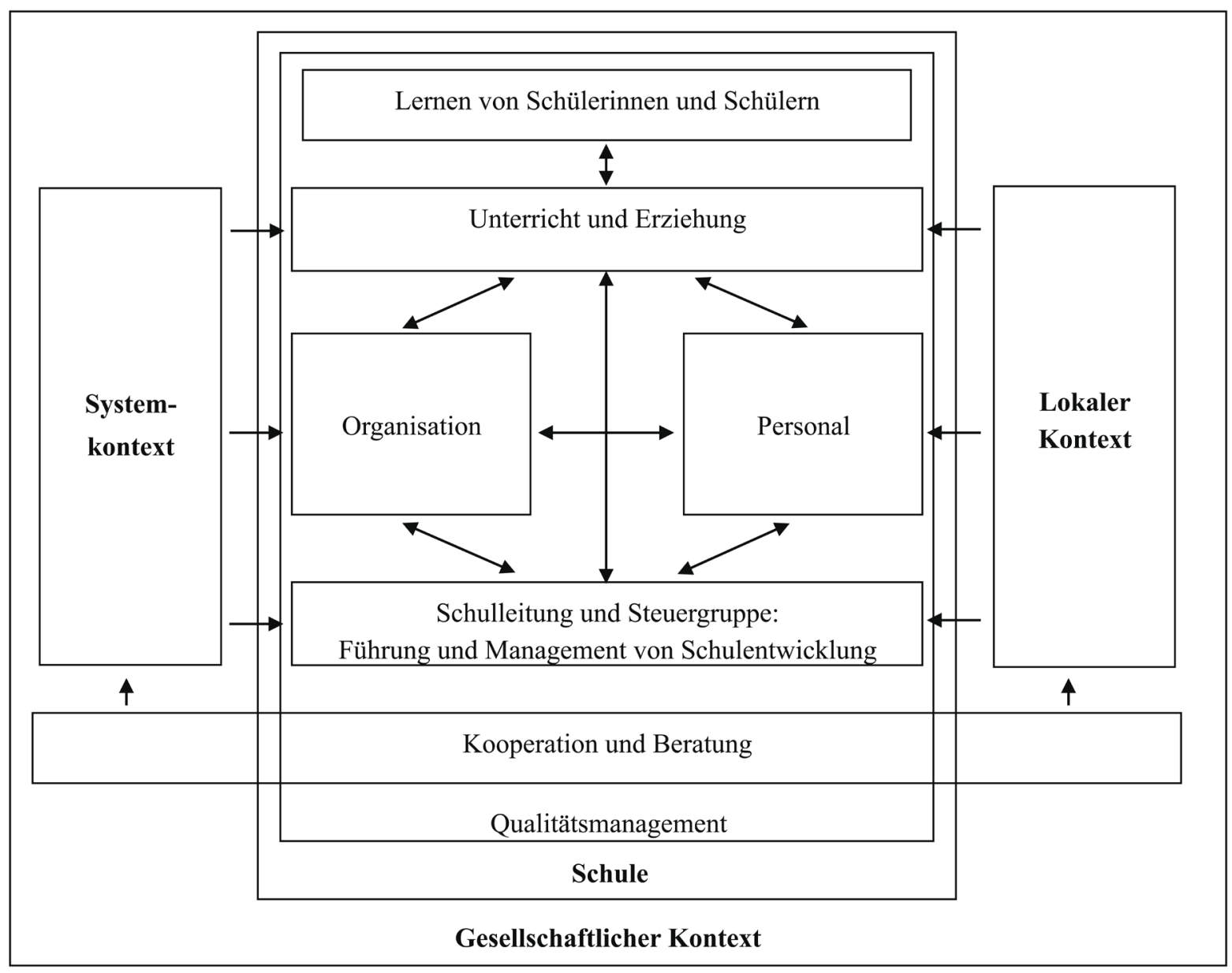

Um die "Wirkungen der Arbeitsbedingungen und Anforderungen auf das Individuum» (Schaper, 2019, S. 386) und somit belastungsrelevante Befunde in dieser Arbeit theoretisch einzuordnen, wird zudem das Rahmenmodell schulischer Belastung (vgl. Böhm-Kasper, 2004) herangezogen. In diesem wird zwischen den drei Ebenen Belastungsfaktoren, subjektiven Deutungsprozessen und Beanspruchung differenziert und deren Wechselwirkungen berücksichtigt (vgl. Abb. 4).

2 Die Handlungsfelder sind in Abb. 3 die nicht hervorgehobenen Aspekte. Im Handlungsfeld «Erziehung» lautet eine Beispieltätigkeit «Ordnungsmassnahmen durchsetzen», im Handlungsfeld «Personal» «dienstliche Beurteilungen vornehmen» etc. (vgl. Huber et al., 2014). 


\section{Abbildung 4}

Rahmenmodell «Schulische Belastung» nach Böhm-Kasper (2004, vgl. auch Huber, 2013)

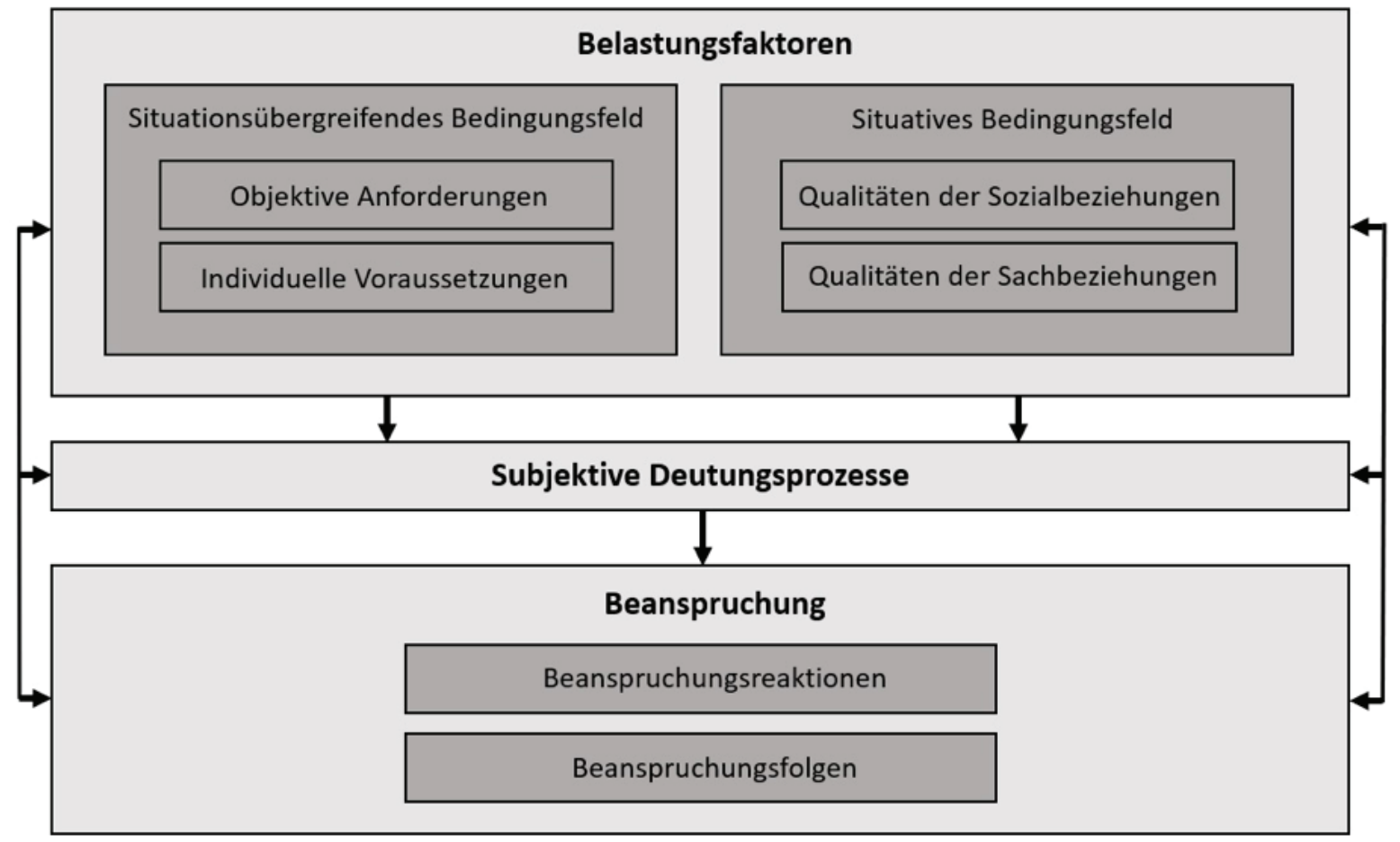

Das Modell unterscheidet dezidiert verschiedene Bedingungen von Belastung, wohingegen etliche weitere Belastungsmodelle aus dem schulischen Kontext stärker intraindividuelle Verarbeitungsprozesse fokussieren, die in dieser Studie nicht im Analysefokus stehen (vgl. im Überblick Dick \& Stegmann, 2013). Zu diesen Belastungsbedingungen wären dann potenziell auch die systembedingten Aufgabenzuschreibungen im Feld der objektiven Anforderungen (vgl. 2.1 und Abb. 4) zu zählen.

\subsection{Rollenanalyse: Schulmanagement und Educational Governance}

«Die Ergänzung [...] durch eine Rollenanalyse erscheint angeraten. Sie hebt die personalen Interaktionsbeziehungen zwischen den Arbeitsplätzen [...] hervor» (Berthel \& Becker, 2013, S. 249). Daher wurde entschieden, die Rollenanalyse anhand der Forschungsperspektive der Educational Governance zu fundieren.

Unter diesem Paradigma wird davon ausgegangen, dass schulische Steuerungsprozesse nicht nur unilateral verlaufen, sondern vielmehr von verschiedenen Akteurskonstellationen in komplexen Mehrebenensystemen beeinflusst werden (Altrichter \& Maag Merki, 2016). Somit wird der Schulleitung auch eine ihr eigene, komplexe und von individuellen und überindividuellen Faktoren abhängige Handlungslogik zugeschrieben, welche nicht ohne Weiteres von aussen direkt gesteuert werden könne (vgl. Bonsen, 2016). Die GovernancePerspektive fokussiert dementsprechend Probleme «unter der Perspektive der Handlungskoordination zwischen verschiedenen Akteuren in komplexen Mehrebenensystemen» (Altrichter \& Maag Merki, 2016, S. 8) und vermag in dieser Arbeitsplatzanalyse zu erklären, weshalb das konkrete Handeln der erforschten Akteursgruppen - insbesondere in ihrer hierarchischen Konstellation (vgl. 2.1) - von den formalen Aufgabenbeschreibungen (potenziell) abweichen kann. Jene Beschreibungen (vgl. 2.1) wären somit als Steuerungsimpuls und Handlungsrahmen zu interpretieren, vor dessen Hintergrund die Akteure ihr konkretes Rollenhandeln in Interaktion mit jeweils anderen Akteursgruppen ausgestalten. Insofern legitimiert diese theoretische Perspektive zusätzlich den Forschungszugriff auf die subjektiven Wahrnehmungen der tatsächlichen Interaktionsbeziehungen und Aufgabenteilungen im Rahmen einer multiperspektivischen Arbeitsplatzanalyse. 


\section{Forschungsstand}

Betrachtet man den Stand der Forschung zum Schulmanagement, so konstatiert Bonsen (2016) ein Theoriedefizit sowie eine starke Fragmentierung, die er durch die situative Spezifität der Einzelschule plausibilisiert. In seinem Überblick über den Forschungsstand formuliert Wissinger (2014) das folgende Desiderat:

Interessant wäre, etwas über objektivierbare und strukturelle Faktoren des Managements einer Schule und der Führung eines Kollegiums zu wissen, um sicherer kalkulieren zu können, welche Bedingungen gegeben sein müssen, damit Schulleitungspersonen im Sinne der Erwartungen der schulpraktischen Schlussfolgerungen der OECD initiierten Outputforschung erfolgreich sein können (ebd., S. 160).

An dieser Stelle können zur Schulleitung nur ausgewählte Befunde kurz skizziert werden. Die für diesen Beitrag beispielhaft und nach Relevanz für die Zielsetzung ausgewählten deutschsprachigen und internationalen Forschungsbefunde sind in Tabelle 1 zusammengestellt.

\section{Tabelle 1}

Übersicht der ausgewählten Befunde und Themen zu Aufgaben und Rahmenbedingungen von Schulleitungen (eigene Darstellung)

\begin{tabular}{|c|c|}
\hline $\begin{array}{l}\text { Komponente des } \\
\text { Schulmanagements }\end{array}$ & Forschungsbefunde und -themen zur Schulleitung \\
\hline Aufgaben & 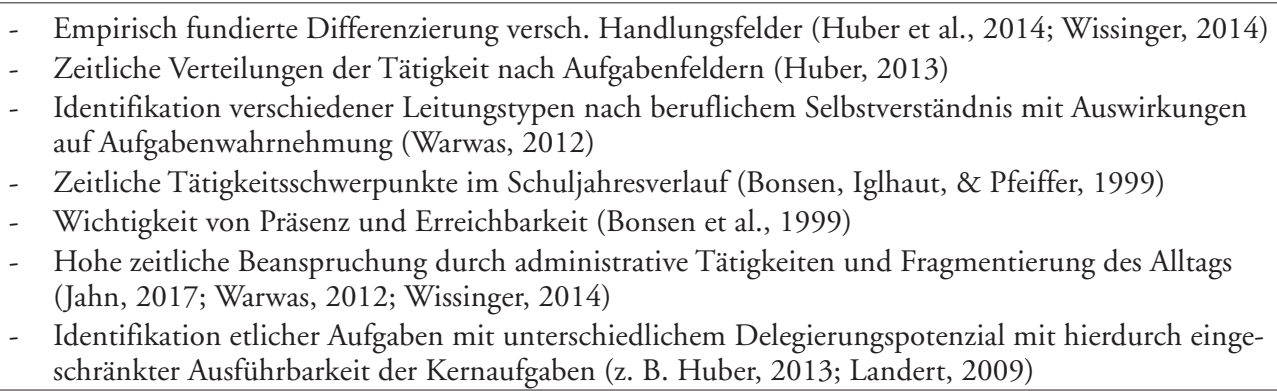 \\
\hline Personen & $\begin{array}{l}\text { - Zusammenstellung von Stressoren von Schulleitung, insb. strukturelle Regulationsbehinderungen } \\
\text { (Dadaczynski \& Paulus, 2015; Warwas, 2012) } \\
\text { - } \quad \text { Zusammenstellung situativer und personaler Ressourcen (Krause et al., 2013) }\end{array}$ \\
\hline Funktion & $\begin{array}{l}\text { - Fehlende oder unzureichende Führung der Schulleitung als potenzielle Struktur und Entlastungs- } \\
\text { funktion (Krause et al., 2013) } \\
\text { - } \quad \text { Inkonsistente Rahmenbedingungen erschweren Rollenklarheit (Jahn, 2017) }\end{array}$ \\
\hline Strukturen & $\begin{array}{l}\text { - Als teilweise unzureichend eingeschätzte Handlungs- und Entscheidungsspielräume (Krause et al., } \\
\text { 2013) } \\
\text { - Einrichtung einer erweiterten Schulleitung mit dann höherer Kooperationsqualität und mehr Zeit } \\
\text { für strategische Aufgaben (Greubel, 2017) }\end{array}$ \\
\hline $\begin{array}{l}\text { Wechselwirkungen } \\
\text { zwischen den } \\
\text { Komponenten }\end{array}$ & $\begin{array}{l}\text { - Schulverwaltungen mit entlastender Funktion bei klarer Aufgabentrennung (Bonsen et al., 1999; } \\
\text { Krause et al., 2013) } \\
\text { - Überstunden als Regelfall (z. B. Dorsemagen, Baeriswyl \& Krause, 2013; Pekruhl et al., 2011) } \\
\text { - } \begin{array}{l}\text { Unzureichende Rahmenbedingungen, insb. Arbeitszeit, im Widerspruch zu hohen Erwartungen an } \\
\text { das Schulleitungsamt (Dadaczynski \& Paulus, 2015; Krause et al., 2013; Wissinger, 2014) }\end{array}\end{array}$ \\
\hline
\end{tabular}

Die Forschungslage zu Schulsekretariaten bzw. Schulverwaltungen ist stark defizitär. Eine quantitativ angelegte, arbeitspsychologische Studie identifiziert Belastungen, Aufgaben, Probleme und Ressourcen an der Schnittstelle Schulsekretariat im Vergleich zu anderen Berufsgruppen mit administrativem Tätigkeitsschwerpunkt. Hierbei werden hohe Werte im Bereich der Arbeitszufriedenheit, gering ausgeprägte Burnout-Indikatoren, geringe soziale Unterstützung (durch Lehrende, Vorgesetzte oder Behörden) sowie teilweise hohe Beeinträchtigungen durch arbeitsplatzfremde Aufgaben bei Mitarbeitenden von Schulsekretariaten hervorgehoben (vgl. Dormann, et al., 2004).

Forschungsergebnisse zu Aufgaben und Rahmenbedingungen im Schulmanagement mit speziellem Bezug auf Schulpflegen liegen in geringem Masse vor, da im deutschsprachigen Raum das Schweizer Milizsystem mit den Schulpflegen (auch: Schulbehörden, Schulkommissionen) eine Sonderrolle einnimmt. Einer der wohl relevantesten Befunde ist, dass ein Grossteil der bei Quesel et al. (2014) befragten Schulleitungen die Schul- 
pflegen/Schulkommissionen durch die Einführung von (hauptberuflichen) Schulleitungen für überflüssig hält. Gleichzeitig werden Rekrutierungsschwierigkeiten der Schulpflege aufgrund erhöhter Anforderungen und Belastungen angeführt und ein erhöhter Professionalisierungsbedarf festgestellt (Dlabac, 2016; Trachsler et al., 2007; Trachsler et al., 2005). Befunde aus einer Analyse von Sitzungsprotokollen deuten darauf hin, dass die Schulpflege eine starke und durchaus funktionale Vertretung lokalspezifischer Interessen der Schule innerhalb der Gemeinde darstellt. Gleichzeitig werde mit der Einführung von Schulleitungen Steuerungsmacht von der Schulpflege an die kantonalen Behörden verlagert und Verantwortung an die Schulleitung delegiert («bypassing», vgl. Heinzer, 2016). Huber (2011a) fasst die Lage wie folgt zusammen: «Within this general re-structuring of the governing in the Swiss cantons, the school governing bodies experience far reaching pressures regarding both their political and educational function» (ebd., S. 482).

Weitere Befunde aus den Studien von Trachsler et al. $(2007,2005)$ deuten darauf hin, dass die Schulpflege neben Organisatorischem vor allem strategische Aufgaben im Bereich Schulentwicklung, Unterrichtsbesuche und Personalführung übernimmt. Seitens der Schulpflegen werden eigene pädagogische Fähigkeiten/Wissensbestände nicht als defizitär wahrgenommen und Schulleitungen wird eine Entlastungsfunktion für Schulpflegen attestiert (Trachsler et al., 2007, 2005).

Es zeigt sich, dass sich nicht nur der Forschungsstand zum Schulleitungshandeln fragmentarisch darstellt, sondern insbesondere auch derjenige zum Schulmanagement unter der Berücksichtigung verschiedener Akteure. Bonsen (2016) spricht in diesem Kontext von der Notwendigkeit einer Theorie des Schulleitungshandelns, um praxisrelevante Handlungs- und Steuerungsempfehlungen auszusprechen. In der Perspektive der Educational Governance, insbesondere unter Berücksichtigung der schweizspezifischen Befunde zur Schulpflege, erscheint eine multiperspektivische Betrachtung des Schulmanagements unter Einbezug zentraler Akteursgruppen umso stärker geboten.

Als weitere Desiderata aus Theorie- und Forschungsstand lassen sich ableiten:

- Ermittlung der Wirkungsweise von Belastungen,

- Ausbau und Kontextualisierung eines empirisch fundierten Aufgabenkatalogs im Schulmanagement,

- Erkenntnisse über objektivierbare und strukturelle Faktoren schulischen Leitungshandelns zur Verbesserung der Arbeits(platz)bedingungen.

\section{Zielsetzung und Forschungsdesign}

Im Fokus der hier konzipierten Arbeitsplatzanalyse steht «die Einschätzung spezifischer Arbeitsmerkmale, -aufgaben und -bedingungen in einem kognitiv interpretierenden bzw. evaluativen Sinne» (Schaper, 2019, S. 395). Damit wird auch auf die Ermittlung «subjektiver Einschätzungen zu Stressoren und Indikatoren des Stresserlebens sowie deren Zusammenhänge» (ebd.) abgezielt. Vor dem Hintergrund der Forschungsperspektive der Educational Governance ist ferner nach der Funktionalität von Handlungskoordination im Schulmanagement zu fragen. Zusammen mit der Konzeptionierung von Schulmanagement und der aus der Empirie (3) abgeleiteten Desiderata lauten daher die Ziel- und Fragestellungen dieses Beitrags:

Die Studie analysiert die Aufgaben und Schnittstellen im Schulmanagement der Volksschule im Kanton Aargau mit all ihren Wechselwirkungen aus der Perspektive der Schulleitungen, Schulverwaltungen und Schulpflegen vor dem Hintergrund kantonaler Rahmenbedingungen:

- Welche Aufgaben werden von welchen der drei Akteuren übernommen und wie lassen sich die Aufgaben strukturieren hinsichtlich Relevanz, Umfang, Spektrum, Funktionalität, Machbarkeit und Belastungserleben?

- Wie sind die Schnittstellen zwischen den drei Akteuren ausgestaltet und wie klar, effizient, funktional, belastend und/oder entlastend ist die Arbeitsteilung?

Das in diesem Beitrag analysierte Datenmaterial entstand im Mai/Juni 2016 im Zusammenhang des Projekts «Arbeitsplatzanalyse Schulleitung Volksschule» im Auftrag des Departements Bildung, Kultur und Sport (BKS) Aargau, durchgeführt vom Institut für Bildungsmanagement und Bildungsökonomie (IBB) der PH Zug.

Die Fragestellung legt eine Erhebung mittels leitfadengestützter Experteninterviews nahe, mit denen subjektive Wahrnehmungen, Deutungen und Wirklichkeitskonzepte der Akteure verstanden, nachvollzogen und analysiert werden können (vgl. Flick, 2014). Sie verfügen als Repräsentierende ihrer Akteursgruppe über spezifisches (Experten-)Wissen bezüglich des hier verfolgten Forschungsinteresses. Als Auswertungsmethode wurde die Qualitative Inhaltsanalyse nach Mayring (2015) gewählt. Das umfassende Forschungsinteresse 
verlangt nach einer guten Strukturierungsleistung der Auswertungsmethode. Dies leistet die inhaltlich-strukturierende Inhaltsanalyse, indem anhand eines theoriebasiert-deduktiven Kategoriensystems spezifische Themen und Aspekte aus dem Datenmaterial extrahiert werden (ebd., S. 103). Mit einer sich anschliessenden zusammenfassenden Inhaltsanalyse wird das dann subsumptionslogisch strukturierte Material sukzessive abstrahiert und weiter zusammengefasst, um «eine grosse Materialmenge auf ein überschaubares Mass zu kürzen und die wesentlichen Inhalte zu erhalten» (ebd., S. 85). Als Kodiereinheit wurde eine sinnlogisch zusammenhängende Interviewpassage ausgewählt. Von Doppelkodierungen wurde abgesehen. Die Auswertungskategorien wurden deduktiv aus den (theoriegenerierten) Fragestellungen in Verbindung mit den Handlungsfeldern im Schulmanagement (2.2) erstellt. Die Kodierlogik ergibt sich damit wie in Tabelle 2 und 3 dargestellt.

Da zu den Handlungsfeldern ausführliche Beschreibungen in Form von Beispieltätigkeiten vorliegen (vgl. Huber et al., 2014), wurden diese deduktiv als Substitut für Kodierregeln verwendet. Bei vereinzelt vorkommenden Trennschärfeproblemen, d. h., wenn die betreffende Kodiereinheit allen oder mehreren Handlungsfeldern (respektive Sub-Sub-Subkategorien) zuzuordnen ist, wurde diese der übergeordneten Kategorie zugewiesen und entsprechend bei der Auswertung berücksichtigt. Die Kategoriendefinitionen im Kodierleitfaden wurden entsprechend formuliert (vgl. Tab. 3). Die Interviewpassagen wurden folglich ausschliesslich in den Sub-Subkategorien oder niedriger kodiert. Mit diesem Vorgehen konnte dem Nachteil begegnet werden, dass aus Machbarkeitsgründen nur eine Person die Kodierung durchgeführt hat und entsprechend auf die Angabe der Intercoderreliabilität verzichtet wird. Zur Nachvollziehbarkeit des Kategoriensystems sind in Tabelle 3 beispielhaft einzelne Kategorien aus dem Kodierleitfaden angeführt.

\section{Tabelle 2}

Übersicht (deduktive) Auswertungskategorien und-ebenen

\begin{tabular}{|c|c|c|c|c|}
\hline $\begin{array}{l}\text { Hauptkate- } \\
\text { gorie } \\
\text { (befragter } \\
\text { Akteur) }\end{array}$ & \multicolumn{2}{|c|}{$\begin{array}{l}\text { Subkategorie } \\
\text { (Forschungs- } \\
\text { frage) }\end{array}$} & $\begin{array}{l}\text { Sub-Subkategorie } \\
\text { (Aspekt der Forschungsfrage) }\end{array}$ & $\begin{array}{l}\text { Sub-Sub-Subkategorie } \\
\text { (Handlungsfeld) }\end{array}$ \\
\hline \multirow{2}{*}{$\begin{array}{l}\text { Schulleitung } \\
\text { (SL)/ } \\
\text { Schulverwal- } \\
\text { tung (SV)/ } \\
\text { Schulpflege } \\
\text { (SP) }\end{array}$} & \multicolumn{2}{|c|}{ Aufgaben } & $\begin{array}{l}\text { Relevanz/Umfang/Spektrum/ } \\
\text { Veränderung/Funktionalität/ } \\
\text { Machbarkeit/Belastung }\end{array}$ & $\begin{array}{l}\text { Erziehung/Personal/Organisation und Verwaltung/ } \\
\text { Qualitätsentwicklung/Qualitätssicherung/Repräsen- } \\
\text { tieren/Kooperation/Eigene Weiterbildung }\end{array}$ \\
\hline & $\begin{array}{l}\text { Schnitt- } \\
\text { stellen }\end{array}$ & $\begin{array}{l}\text { SL/ } \\
\text { SV/ } \\
\text { SP }\end{array}$ & $\begin{array}{l}\text { Aufgaben/Klarheit der Aufgaben- } \\
\text { teilung/Effizienz der Schnittstelle } \\
\text { /Funktionalität/Belastung }\end{array}$ & $\begin{array}{l}\text { Erziehung/Personal/Organisation und Verwaltung/ } \\
\text { Qualitätsentwicklung/Qualitätssicherung/Repräsen- } \\
\text { tieren/Kooperation/Eigene Weiterbildung }\end{array}$ \\
\hline
\end{tabular}

Tabelle 3

Definition exemplarischer Kategorien gemäss dem Kodierleitfaden (nur Hauptkategorie Schulleitung)

\begin{tabular}{l|l|l}
\hline Kategorie & Definition & Ankerbeispiel \\
\hline SL-Aufgaben-Relevanz & $\begin{array}{l}\text { Einschätzung der Schulleitung zu unter- } \\
\text { schiedlicher Relevanz der Aufgaben, } \\
\text { Benennung dieser Aufgaben. Aufgaben, } \\
\text { die alle oder mehrere Handlungsfelder } \\
\text { umfassen oder darüber hinausgehen. }\end{array}$ & $\begin{array}{l}\text { Laden irgendwie kann zusammenhalten, oder? Es gibt } \\
\text { nicht ein bestimmtes Gebiet, wo ich sagen kann, das ist } \\
\text { mein wichtigstes, das kann ich nicht sagen» (SL7). }\end{array}$ \\
\hline $\begin{array}{l}\text { SL-Aufgaben-Relevanz- } \\
\text { Handlungsfeld Personal }\end{array}$ & $\begin{array}{l}\text { Einschätzung der Schulleitung } \\
\text { zur Relevanz der Aufgaben im } \\
\text { Handlungsfeld Personal, auch: } \\
\text { Benennung dieser Aufgaben. }\end{array}$ & $\begin{array}{l}\text { "Bezogen auf SOLL ist natürlich das ganz grosse } \\
\text { Kernthema Personalführung bei uns. Also Zeit haben } \\
\text { für Lehrpersonen, bei mir gehört dazu der Hausdienst } \\
\text { noch dazu, Sekretariat gehört dazu, Schulsozialarbeit } \\
\text { gehört dazu. Da müsste ich eigentlich den grössten Teil } \\
\text { meiner Zeit und meiner Energie investieren können» } \\
\text { (SL4). }\end{array}$ \\
\hline $\begin{array}{l}\text { SL-Schnittstellen-SP- } \\
\text { Belastung }\end{array}$ & $\begin{array}{l}\text { Aussagen aus der Perspektive der Schul- } \\
\text { leitung zur Schnittstelle mit der Schul- } \\
\text { pflege: diesbezügliche Einschätzung } \\
\text { zum Belastungserleben. Aufgabenunab- } \\
\text { hängige Aussagen oder Aussagen, die alle } \\
\text { oder mehrere Handlungsfelder umfassen } \\
\text { oder darüber hinausgehen. }\end{array}$ & $\begin{array}{l}\text { "Was mich belastet mit der Schulpflege ist die Situation } \\
\text { mit dem einen Mitglied. Er [...] lässt uns in dem Sinne } \\
\text { eigentlich gar nicht sachlich bedingt sind, sondern } \\
\text { Gespräch kommt» }\end{array}$ \\
\hline
\end{tabular}


Bei der Stichprobenauswahl handelt sich um ein theoriegeleitetes Sampling, bei welchem aus Theorie und Forschungsstand abgeleitete Heterogenitäts- und Funktionalitätsaspekte des Schulmanagements berücksichtigt werden konnten: Es wurden Schulleitungen von 217 Volksschulen, 200 Schulsekretariaten der jeweiligen Volksschule und 217 Schulpflegegremien der Gemeinden des Kantons Aargau zur Teilnahme an den Interviews eingeladen. 101 Schulleitungen, 40 Schulverwaltungspersonen und 33 Mitglieder der Schulpflege waren bereit, an einem Interview teilzunehmen. Daraus wurde eine möglichst heterogene Stichprobe zusammengestellt. Um die zentrale Stellung der Schulleitungen im Schulmanagement zu berücksichtigen, wurde der Schwerpunkt auf Schulleitungen (8 Personen), nachgelagert auf Interviews mit Mitgliedern der Schulverwaltungen (5 Personen) und Schulpflegen $(5$ Personen) gelegt. Die Akteure der Stichprobe $(n=18)$ verteilen sich in etwa gleich über Volksschulen verschiedener Grössen (zwischen 100-200 bis hin zu über 800 Schüler/-innen) und zugehöriger Schulformen. Dass sich die Stichprobe auch durch eine für die Arbeitsplatzanalyse bedeutsame Spannbreite des Stellenpensums auszeichnet, zeigt Tabelle 4.

Tabelle 4

Darstellung der Stichprobe nach Stellenpensum

\begin{tabular}{l|l|l}
\hline \multirow{2}{*}{$\begin{array}{l}\text { Pensum } \\
(\%)\end{array}$} & Anzahl interviewter Personen \\
\cline { 2 - 3 } & SL & SV \\
\hline $0-19$ & 0 & 0 \\
\hline $20-39$ & 2 & 1 \\
\hline $40-59$ & 2 & 1 \\
\hline $60-79$ & 2 & 1 \\
\hline $80-100$ & 2 & 1 \\
\hline k. A. & 0 & 1 \\
\hline
\end{tabular}

Bem. $. \mathrm{SL}=$ Schulleitung, SV = Schulverwaltung; k.A. = keine Angabe

\section{Ergebnisse}

Die Ergebnisse werden zunächst entlang der Analysekomponenten der Arbeitsplatzanalyse (vgl. 2) und abschliessend in einer übergreifenden Betrachtung belastungsrelevanter Befunde (5.3) dargestellt. Dies geschieht in Form abstrakter Zusammenfassungen entlang der Haupt- und Subkategorien als Resultat der zusammenfassend-strukturierenden Inhaltsanalyse. Somit können jeweils anschliessend aus theorie- und empiriegeleiteten Argumentationen die wichtigsten Erkenntnisse dieser Arbeit als Thesen gebündelt abgeleitet werden.

\subsection{Ergebnisse der Arbeitsanalyse}

Es lassen sich folgende akteurspezifische Schwerpunkte der tatsächlichen Aufgabenübernahme (Subkategorie: Aufgaben; ohne Belastung, vgl. 5.3) identifizieren:

Die Schulleitungen fokussieren nach eigener Auskunft zunächst die Sicherstellung des operativen Tagesgeschäfts, v. a. in den Handlungsfeldern Personal und Organisation/Verwaltung sowie Erziehung. In diesen wird auch der höchste Arbeitsumfang angegeben. Sofern der Schulalltag sichergestellt ist, kann gemäss eigenen Aussagen nachrangig Personal-, Unterrichts- und Qualitätsentwicklung betrieben werden:

Und deshalb ist für mich ganz klar, dass die Organisation des Schulbetriebs Hauptaufgabe Nummer eins ist für mich. Und erst wenn das einigermassen klappt, hat man Zeit für andere Dinge. [...] Die da wären, beispielsweise Qualität in der Schule, beim Unterrichten, ja, eben auch Unterrichtsbesuche, Feedbacks geben, den Lehrpersonen, mit ihnen neue Dinge anziehen. Mit ihnen die Schule zu verbessern zu versuchen. Aber zuerst muss das Ganze laufen (SL5).

Hinsichtlich der Machbarkeit der Aufgaben geben Schulleitungen die hohe Fragmentierung, Unplanbarkeit und Vielfalt an Aufgaben im Arbeitsalltag als effizienzhinderlich an:

Den alläglichen Wahnsinn, dass es nie so läuft wie man plant. Weil permanent irgendwie E-Mail reinkommt, ein Anruf reinkommt, irgendwo etwas nicht rund läuft, zwei Knatsch haben miteinander. Und dann die Themen eigentlich vorgegeben werden vom Alltag. Ich habe schon einen Plan, jeden Tag, was ist mein Hauptthema - das klappt selten bis nie (SL4).

Vor allem Aufgaben im Bereich Qualitätsentwicklung, insbesondere Unterrichts- und Personalentwicklung, werden aus Machbarkeitsgründen von den Schulleitungen nachrangig betrachtet (s. o.): 
Wenn immer die Sachen, die eigentlich auch wichtig wären, unter den Tisch gekehrt werden, wie die Klassenbesuche, Mitarbeitergespräche, wozu man einfach nicht kommt, dann hat das schlussendlich enorme Auswirkungen auf die Qualität (SLG).

Dass die Sicherstellung des Schulalltags durch die Bearbeitung von Aufgaben in den Handlungsfeldern Personal und Organisation/Verwaltung sowie Erziehung als am umfangreichsten sowie als die erste Priorität der Tätigkeit von Schulleitungen genannt wird, kann als zentraler, neuer Befund eingeordnet werden. Verknüpft man diesen mit der Theorie zum Schulmanagement (2.2), liegt der folgende Schluss nahe:

Die aus Akteursperspektive bestimmende Aufgabe im Alltag des Schulmanagements im untersuchten Feld ist nicht die «Gestaltung und Optimierung von Schule und schulischen Prozessen» (Huber, 2011b, S. 2). Vielmehr kann der Schwerpunkt des "professionelle[n], also qualifizierte[n], systematische[n] und zielgerichtete[n] Management[s] der Arbeit von Schule» (ebd.) im Sinne einer Sicherstellung und Ermöglichung dieser Arbeit hervorgehoben werden. Dass jenes Management realiter in systematischer Weise geschieht, erscheint aufgrund der von den Akteuren geschilderten Fragmentierung der Aufgaben und ihrer Unplanbarkeit sowie dem Wunsch nach Ungestörtheit und Fokussierungsmöglichkeit während der Arbeitszeit zweifelhaft (vgl. hierzu Jahn, 2017; Warwas, 2012). Aus der Perspektive der befragten Schulleitungen kann angesichts dieser Ergebnisse formuliert werden:

These 1: Aufgaben in den Bereichen Qualitäts-, Personal- und Unterrichtsentwicklung werden im Schulmanagement im untersuchten Feld nicht angemessen berücksichtigt. Die Sicherstellung des Schulalltags geniesst aus Sicht zentraler Akteure die höchste Priorität.

Auch aus weiteren Forschungsbefunden lässt sich diese These stützen: Durch nicht delegierbare Aufgaben wird die verfügbare Zeit für die Hauptaufgaben der Schulleitung stark eingeschränkt, eine zeitliche Überbeanspruchung ist der Normalfall (Dorsemagen et al., 2013; Huber, 2013) und die Rahmenbedingungen des Arbeitsplatzes Schulleitung entsprechen nicht den hohen Erwartungen an das Handeln im Schulmanagement (Wissinger, 2014).

Die Schulpflege entlastet nach eigenen Angaben als höhere Instanz die Schulleitung bei Konfliktfällen in den Handlungsfeldern Erziehung und Personal und ist für Anstellungen und Entlassungen zuständig. Diese Aufgaben werden von den Schulpflegen auch als die umfangreichsten und wichtigsten charakterisiert. Daneben trifft die Schulpflege Entscheidungen im Handlungsfeld Qualitätsentwicklung.

Angesichts der in der offiziellen Aufgabenbeschreibung prominent zugeteilten Aufgabe der strategischen Führung (2.2) erscheint diese Gewichtung bemerkenswert. Hier kann die Rollenanalyse der Handlungslogik in der Schnittstelle Schulleitung-Schulpflege Erklärungshinweise liefern (s. u.). Mit Verweis auf Forschungsbefunde zur Professionalisierungsbedürftigkeit der strategischen Aufgabe der Schulpflege (Dlabac, 2016; Trachsler et al., 2007, 2005) lässt sich eine angemessene Berücksichtigung des Handlungsfeldes Qualitätsentwicklung sowie von Aufgaben in Unterrichts- und Personalentwicklung zumindest anzweifeln (vgl. These 1).

Die Schulverwaltung übernimmt hauptsächlich Aufgaben im Handlungsfeld Organisation/Verwaltung. Priorität geniessen dabei lohnrelevante Vorgänge im administrativen Tätigkeitsfeld Personal, als umfangreich werden v. a. Aufgaben im Kontext der Schulpflegesitzungen (Protokollierung, Folgeaufträge) angegeben. Machbarkeitsprobleme entstehen analog zur Schulleitung bezüglich hoher Fragmentierung und partieller Unplanbarkeit von Aufgaben.

Sowohl Umfang als auch Relevanz von Aufgaben werden von allen Akteursgruppen als abhängig vom Schuljahresverlauf angegeben, was sich mit bisherigen Forschungsergebnissen deckt (vgl. Bonsen et al., 1999). Ebenso äussern alle Akteure bezogen auf die Veränderung ihrer Aufgaben in den letzten Jahren einen Anstieg in Komplexität und Umfang, insbesondere trifft dies auf die Aussagen von Schulleitungen und Schulpflegen im Konfliktmanagement mit Eltern zu. Vergleicht man die genannten Aufgabenschwerpunkte, so zeigt sich als Gemeinsamkeit auch der zeitliche Fokus auf Tätigkeiten in den Handlungsfeldern Organisation/Verwaltung, Erziehung und Personal, der in bisheriger Forschung zur Schulleitung ebenfalls identifiziert wurde (Huber, 2013; Wissinger, 2014).

\subsection{Ergebnisse der Rollenanalyse}

Die Ergebnisse ausgehend von den Aussagen der Akteure in der Subkategorie «Schnittstellen» stellen sich wie folgt dar: 


\subsubsection{Innerhalb einer Akteursgruppe}

Bezogen auf die schulleitungsinterne Schnittstelle (Subkategorie SL-Schnittstellen-SL) waren bei insgesamt sechs der acht interviewten Schulleitungen weitere Schulleitungspersonen an der Volksschule der jeweiligen Gemeinde vorhanden. Organisations- bzw. Hierarchiemodelle, die Aufteilung des Stellenpensums und die Arbeitsteilung innerhalb der Schulleitung gestalten sich jeweils unterschiedlich und sind nicht kantonal festgelegt (vgl. 2.1). Auf Basis der Interviewdaten konnten vornehmlich zwei unterschiedliche Leitungsmodelle identifiziert werden (Abb. 5).

Die Zuständigkeiten im einstufigen Leitungsmodell werden von den Schulleitungen nach eigenen Auskünften zumeist nach Schulhäusern aufgegliedert (sogen. Schulhausleitungen), teilweise sind diese auch zusammengelegt, sodass eine Schulleitungsperson die Schulhausleitung von mehreren Schulhäusern bzw. Schulstufen innehat. Die Schulleitungen, die in diesem Organisationsmodell arbeiten, führen in allen Handlungsfeldern eigenständig ihr jeweiliges Schulhaus. Aufgaben in Zusammenarbeit mit den anderen Schulhausleitungen liegen vor allem im Bereich Qualitätsmanagement und -sicherung sowie Kooperation nach aussen und Organisation/Verwaltung. Hier existiert ein reger Informationsaustausch und die Aufgabenteilung wird zumeist als klar eingeschätzt.

In einem zweistufigen Leitungsmodell gibt es noch eine den Schulhausleitungen vorgesetzte Gesamtschulleitung, die möglicherweise auch gleichzeitig eine Schulhausleitung ist. Diese ist meist für das Fachlehrpersonal und das Qualitätsmanagement zuständig. Die Funktionalität wird in den meisten Fällen als gut angesehen, Unklarheiten in der Arbeitsteilung werden nicht geäussert.

Abbildung 5

Identifizierte Leitungsmodelle Volksschule (eigene Darstellung)

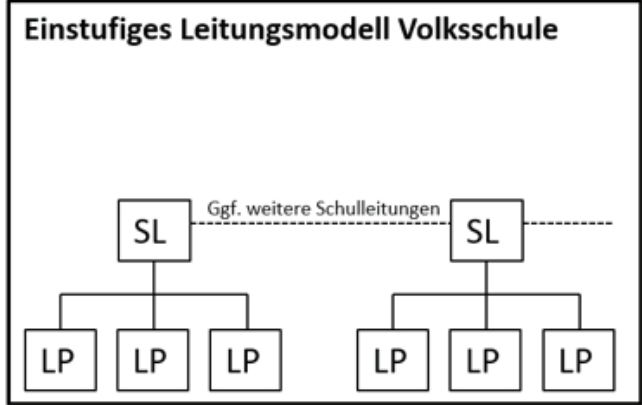

GSL: Gesamt-Schulleitung

SL: Schulleitung

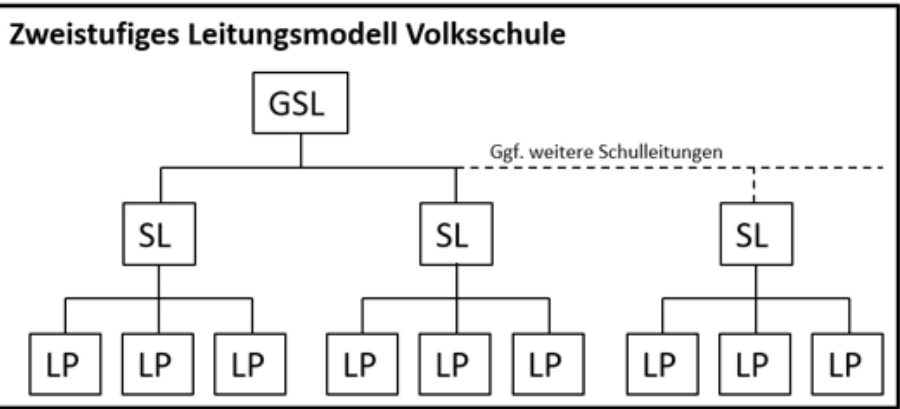

LP: Lehrperson

Offenbar überführen die lokalen Akteure die kantonalen Vorgaben zum Stellenpensum der Schulleitung (vgl. 2.1) im Sinne eines Steuerungsimpulses gemäss eigener Einschätzung in lokalspezifisch ausgestaltete Hierarchiestrukturen. Dies ist bemerkenswert, denn so werden Rahmenvorgaben seitens der Makroebene auf spezifische Art und Weise folgenreich auf der Mesoebene performiert:

These 2: Im untersuchten Feld wird das hierarchische Leitungsmodell der Volksschule innerhalb kantonaler

Rahmenbedingungen lokalspezifisch ausgestaltet, wovon das Aufgabenspektrum und das zur Verfügung stehende

Stellenpensum der einzelnen Schulleitungsperson(en) zentral mitbestimmt wird.

Wie (und von wem) dies geschieht, kann mit dem hier vorliegenden Datenmaterial nicht beurteilt werden. Wohl aber können die Einflüsse dieser Hierarchiemodelle auf das subjektive Erleben der Interaktions- und Kooperationsqualität analysiert werden:

Im einstufigen Leitungsmodell liegt eine geäusserte Belastung in fehlender Kongruenz des Rollenverständnisses bei mehr als drei Schulleitungspersonen mit kleinem Pensum. Hier konstatiert die Schulleitung Effizienzverluste und wiederkehrende Konflikte sowie unklare Zuständigkeiten im Bereich Qualitätsmanagement, welche durch eine Gesamtschulleitung lösbar seien. Als Entlastung wird vor allem im zweistufigen Modell die Tatsache genannt, von einem etwaigen Erfahrungs- oder Wissensvorsprung der Schulleitungskolleginnen und -kollegen profitieren zu können und stets Ansprechpartner/-innen mit ähnlicher Funktion zu haben.

Immer dort, wo ich unsicher bin oder gerne eine Zweitmeinung habe, habe ich mindestens zwei Kollegen, die ich fragen kann. Das finde ich sehr entlastend (SL6). 
Als problematisch und belastungsintensiv wird die schulleitungsinterne Schnittstelle im zweistufigen Modell nur dann beschrieben, wenn mehrere Schulleitungen mit geringem Pensum an derselben Volksschule arbeiten.

Wir hatten früher noch ein Schulleitungsmodell gehabt mit Gesamtschulleitung und da hatten wir dann [mehrere] Leute unten dran. Und die haben wir zum Glück jetzt auf [weniger Schulleitungspersonen] reduzieren können. [...] Also Schulleitungspersonen, die hochprozentig angestellt sind, und hochprozentig vor Ort. Das hat es eigentlich leichter gemacht (SL7).

Diese Ergebnisse lassen den Schluss zu, dass eine schulhausbezogene Aufteilung der Schulleitungspersonen, insbesondere ohne eine übergeordnete Gesamtschulleitung, als suboptimal bezüglich der Handlungskoordination eingeschätzt werden kann. Wenn für jede Schulhausleitung eine Schulleitungsperson zugeteilt ist, so führt dies zu einer nachteiligen, ineffizienten und dysfunktionalen Zerstückelung des zur Verfügung stehenden Gesamtpensums der Schulleitungsfunktion durch einen erhöhten Kooperationsaufwand. Die Ergebnisse lassen auf folgende These schliessen:

These 3: Eine Verteilung des zur Verfügung stehenden Gesamtpensums der Schulleitungsfunktion für die jeweilige Volksschule auf möglichst wenige Personen, welche mit möglichst hohen Stellenprozenten angestellt sind, führt zu einer Belastungsreduktion, Motivationssteigerung sowie zu Effizienz- und Funktionalitätsvorteilen.

Für die schulverwaltungsinterne Schnittstelle (Subkategorie SV-Schnittstellen-SV) wurden unterschiedliche Modi der Aufgabenteilung geäussert - mit einer klaren Trennung der Aufgabenbereiche und mit überlappenden Aufgabengebieten. Bei mehreren Mitarbeitenden wird hier ein Bedarf und Wunsch nach einer zusätzlichen, koordinierenden und übergeordneten Hierarchiestufe geäussert.

Dieser Wunsch ergibt sich kongruent auch bei einstufigen Organisationsmodellen der Schulleitung (s. o.). Es liegt die Vermutung nahe, dass durch eine Gesamtschulleitung bzw. eine Schulverwaltungsleitung in grösseren Teams (ab ca. 4 Personen) die genannten Belastungen reduziert und Entlastungen, Funktionalitäten und die Arbeitseffizienz durch die Reduktion bzw. Lösung von Konflikten gefördert werden können (vgl. Greubel, 2017). Somit liesse sich die Handlungskoordination insgesamt optimieren:

These 4: Eine fehlende Gesamtschul- bzw. Schulverwaltungsleitung in grösseren Teams führt zu Konflikten im Bereich der Handlungskoordination, welche sich negativ auf das Belastungserleben sowie die Arbeitseffizienz auswirken.

Die schulpflegeinterne Schnittstelle (Subkategorie SP-Schnittstellen-SP) wird in fast allen Fällen durch die Aufteilung von Ressorts geregelt, welche grösstenteils als klar empfunden wird. Dies deckt sich mit den Befunden von Trachsler et al. (2005, 2007). Diese Schnittstelle wird aufgrund des unterschiedlichen Engagements der Mitglieder als personenabhängig beschrieben.

\subsubsection{Zwischen den Akteursgruppen}

Die Schnittstelle zwischen der Schulleitung und der Schulverwaltung wird seitens der Schulleitungen als sehr wichtig, funktional und entlastend beschrieben. Die Aufgaben in der Schnittstelle lassen sich fast ausschliesslich dem Handlungsfeld Organisation/Verwaltung zuordnen. Sowohl einige Schulleitungen als auch viele der Schulverwaltungen sehen weiteres Entlastungs- und Delegationspotenzial, welches vor allem aufgrund des (geringen) Pensums der Schulverwaltung oder gering ausgeprägter Delegationskompetenz der Schulleitung nicht genutzt wird:

Aber ich höre das auch von anderen Orten. Also dass die Schulverwaltung, die eigentlich SEHR vieles abfedern kann, eine massive Rolle spielt, wie die ausgestattet ist. Und jetzt in meinem Fall, 30\%, können Sie sich ja ausrechnen, das sind zwei Vormittage, [...] wenn an den übrigen Tagen etwas ist, das endet dann meistens bei $\operatorname{mir}(\mathrm{SL2})$.

Also unsere Schulleitung könnte mega viele Sachen noch delegieren. So administratives Zeug. [...] Da denke ich mir, meine Güte, das musst du nicht machen. Also das ist jetzt wirklich nicht Schulleiterarbeit. Dafür verdient der viel zu viel (SV2).

Nur dort, wo das Schulsekretariatspensum über die kantonale Richtlinie hinaus von den Gemeinden aufgestockt wurde, wurde von keinem weiteren Delegationspotenzial berichtet.

Der Forschungsbefund, dass die Entlastungsfunktion der Sekretariate vor allem bei klarer Aufgabenteilung gegeben ist (Bonsen et al., 1999), kann hier angereichert werden: Mitarbeitende der Schulsekretariate berichten von Belastungen und Ineffizienzen, sofern gewisse Aufgabengebiete nicht vollständig oder nicht zusammen mit der vollen Verantwortung an sie delegiert werden und es zu einem erhöhten Kontroll- und Ausbesserungsaufwand auf beiden Seiten kommt. Zusätzlich erscheint bedeutsam, dass entgegen der formalen Struktur Schulleitungen teilweise Aufgaben der Schulverwaltungen übernehmen, sofern letztere nicht mit ausreichend 
zeitlichen Ressourcen ausgestattet sind. Dies wird insbesondere von Akteuren an kleineren Schulen im untersuchten Feld angeführt.

Unter Berücksichtigung der hier kongruenten Perspektiven von Schulleitungen und Schulverwaltungen führt dies zu folgender Erkenntnis:

These 5: Schulverwaltungen haben bei klarer und möglichst umfassender Aufgabendelegation eine hohe Entlastungsfunktion für die Schulleitungen. Insbesondere an kleinen Schulen mit geringem Schulverwaltungspensum besteht weiteres Delegations- und Entlastungspotenzial, welches durch eine Pensenerhöhung abgerufen werden kann. Durch eine klare Aufgabenteilung und ein hohes Bewusstsein für die Zuständigkeiten des Sekretariats kann dieses Entlastungspotenzial auch an Schulen mit höherem Pensum stärker genutzt werden.

Zur Schnittstelle zwischen der Schulleitung und der Schulpflege ist aus der Perspektive der Schulleitungen (zunächst also: Subkategorien SL-Schnittstellen-SP) das Bild unterschiedlich. Teilweise verlangt die Schulpflege einen vertieften Einblick und ein Mitspracherecht, auch bei operativen Tätigkeiten. Dies wird von den Schulleitungen als belastend und einschränkend, sowie ineffizient und dysfunktional beschrieben. In anderen Fällen besteht ein nur sehr geringer Kontakt, hier kommt die Schulpflege vor allem ihren formal zugesprochenen Pflichten nach, zudem sind auch diese partiell an die Schulleitungen delegiert. Vor allem seitens der Schulleitungen wird ein hoher Gestaltungsfreiraum angegeben:

Ja, also es ist so, dass ich normalerweise das Schulprogramm mache. [...] Ich weiss, wann kommt was vom Kanton, ich habe Ahnung, ich weiss auch, in welche Richtung ich die Schule führen will. Und das Schulprogramm stelle ich auch selbstverständlich der Schulpflege vor [...]. Aber da kommt nicht die Schulpflege zu mir und sagt: Wir wollen die Schule strategisch dahinführen. Das mache ich. Und eigentlich bis jetzt, hat sie mir immer gefolgt, hat sie das unterstützt. [...] Also, wenn sie es anders machen würden, wäre es für mich auch wirklich schwierig (SL7).

Gemäss den Schulleitungen unterstützt die Schulpflege vor allem im Konfliktmanagement und in Schulanliegen gegenüber dem Gemeinderat (analog siehe Heinzer, 2016).

Die Schulpflegen (Subkategorie SP-Schnittstellen-SL) benennen die Trennung von operativen und strategischen Aufgaben (vgl. 2.1) und Tätigkeiten als hoch relevant für eine gute Zusammenarbeit mit der Schulleitung. Gleichzeitig charakterisieren sie diese Trennung als nicht immer eindeutig. Dies spiegelt sich in den Ausführungen der Schulleitungen zu Eingriffen in operative Tätigkeiten wider: Hier scheint durch die Trennung strategischer und operativer Führung in der formalen Aufgabenbeschreibung als Rahmenvorgabe (2.1) keine eindeutige Rollenklarheit vorzuliegen, die sich auch in der Interaktion an der Schnittstelle nicht konsequent klären lässt. Zusätzlich wird auf beiden Seiten angemerkt, dass die Trennung trotz schriftlich festgehaltener Aufgabenteilung (Funktionendiagramm o. Ä.) nicht immer eindeutig zu vollziehen ist, was zu Klärungsbedarf sowie zu Konflikten führen kann. Analog erschweren gemäss Jahn (2017) inkonsistente Rahmenbedingungen die Rollenklarheit der Schulleitungen, somit kann hier formuliert werden:

These 6: Die formale Trennung strategischer und operativer Führung schafft keine Rollenklarheit und erschwert Handlungskoordination: Überschreitet die Schulpflege ihre formal zugesprochenen Kompetenzen im Sinne eines Eingriffs in die operative Leitungsebene, so führt dies zu dysfunktionalem und für die Schulleitungen zu belastendem Handeln im Schulmanagement.

Insofern erscheint nachvollziehbar, wenn die Aufgabendelegation und das Vertrauen in die Ausführung von Aufgaben durch die Schulleitung als graduell sehr unterschiedlich und die Schnittstelle von den Akteuren als stark personenabhängig beschrieben werden.

Dies trifft nach Angaben von Schulleitungen und Schulverwaltungen auch auf den Belastungsgrad der Schnittstelle mit der Schulpflege zu. Dieser wird abhängig von den schulbezogenen Kompetenzen, den kommunikativen Fähigkeiten und dem geforderten Einblick in die Schule des jeweiligen Mitglieds der Schulpflege beschrieben. Persönliche Verflechtungen (eigene Kinder an der Schule) der Schulpflegemitglieder werden ebenfalls hierzu angeführt. Beides führt zu der Erkenntnis, dass Belastungen in der Schnittstelle mit der Schulpflege vor allem im situativen Bedingungsfeld, d. h. in den Qualitäten der Sozial- und Sachbeziehungen (vgl. Böhm-Kasper, 2004), zu verorten sind. Wird dies mit den Studien von Trachsler et al. (2005, 2007) und Dlabac (2016) zum Professionalisierungsbedarf der Schulpflegen in Verbindung gebracht sowie mit dem benannten Verweis, dass die zentralen Ideen zur Schulstrategie von der Schulleitung ausgearbeitet werden, so liegt die folgende These nahe:

These 7: Im Handlungsfeld Qualitätsentwicklung besteht die Tendenz, dass das formale Führungsverhältnis umgekehrt wird und die Schulleitung die Schulpflege gewissermassen ,von unten' führt: Aufgrund eines Professionalisierungsvorsprungs und der unmittelbareren Nähe zur Schule werden tendenziell zentrale Entscheidungen bezüglich der Schulstrategie de facto von der Schulleitung gefällt. 
Auch die angeführte Motivation von Schulleitungen im Handlungsfeld Qualitätsentwicklung sowie der Befund von Krause et al. (2013), dass Schulleitungen in der Schweiz ihre Handlungs- und Entscheidungsspielräume als unzureichend ansehen, unterstützen diese These. Zudem lassen sich hierdurch die Befunde von Heinzer (2016) zum «bypassing» der Schulpflege im Steuerungsgefüge zwischen Kanton und Schulleitung illustrieren.

Die Schnittstelle zwischen der Schulverwaltung und der Schulpflege ist gemäss den Aussagen beider Akteursgruppen durch einen geringen Grad an Zusammenarbeit bzw. gemeinsamen Aufgaben gekennzeichnet.

Im Sinne der Rollenanalyse aus der Perspektive der Educational Governance betrachten wir abschliessend die Thesen 2 bis 7 vor dem Hintergrund der Komponenten des Schulmanagements. Es zeigt sich, in welcher Vielfalt und Intensität die relevanten Personen im Sinne einer Handlungskoordination mehrerer Akteure an Schnittstellen im Schulmanagement auf die Ausführung konkreter Aufgaben einwirken:

Also 50\% von meiner Tätigkeit ist Schnittstellentätigkeit (SL7).

Es kann somit als übergeordneter, schnittstellenbezogener Forschungsbefund angeführt werden:

These 8: Schulisches Leitungshandeln im untersuchten Feld ist in hohem Masse beeinflusst von der institutionell, lokal und individuell geprägten Ausgestaltung der relevanten Schnittstellen und Handlungskoordination im Schulmanagement. Diese These betrifft sowohl Aufgabenart und -umfang als auch das Belastungs- und Entlastungserleben sowie die Funktionalität und Effizienz des Handelns.

\subsection{Ergebnisse zu Belastungen aus Arbeits- und Rollenanalyse}

Abschliessend gilt es nun, belastungsrelevante Befunde beider Analysekomponenten der Arbeitsplatzanalyse vergleichend in das Rahmenmodell schulischer Belastung einzuordnen. Zu diesem Zweck werden zunächst die akteurspezifischen Ergebnisse zur aufgabenbezogenen Belastung (Subkategorie: Aufgaben; Sub-Subkategorie: Belastung) dargestellt:

Aufgabenbezogene Belastungsschwerpunkte werden von Schulleitungen verstärkt in den Handlungsfeldern Erziehung und Personal im Konflikt- und Krisenmanagement und bei kurzfristigem Personalausfall geäussert. Meinungsverschiedenheiten werden für gewöhnlich nicht als belastend erlebt, aber es ergeben sich durchaus aufgabenbezogene Belastungen in der Interaktion:

Also dann, wenn es menschlich, also wenn etwas im Weg ist von der Art von der Person, die aber massgebend wichtig ist für meinen Auftrag, dann ist es schwierig (SL7).

Schulpflegen geben an, nur geringfügig durch ihre Aufgaben belastet zu sein, vornehmlich im Konfliktmanagement bei Elterngesprächen. Auch Schulverwaltungen äußern nur wenig aufgabenspezifische Belastungen, diese entstehen durch potenzielle Loyalitätskonflikte zwischen Schulleitung und Lehrpersonen.

Zusammen mit den schnittstellenbezogenen Erkenntnissen aus der Rollenanalyse (insb. Thesen 4, 6 und 8) liegt mit Bezugnahme auf Böhm-Kasper (2004) die folgende Interpretation nahe:

These 9: Im untersuchten Feld liegen aufgabenbezogene Belastungsfaktoren im Schulmanagement i. A. nicht in den Qualitäten der Sachbeziehungen, sondern eher in den Qualitäten der Sozialbeziehungen (personenbezogene Konfliktlagen), objektiven Anforderungen (hoher Umfang und grosse Menge der Aufgaben in Verbindung mit begrenzten zeitlichen Ressourcen, Personalausfall) oder in deren Zusammenwirken begründet.

Diese These lässt sich durch die Auflistung von Dadaczynski und Paulus (2015), bei Warwas (2012) sowie mit den Befunden für die Schweiz (Huber, 2013, vgl. Tab. 1) stützen.

\section{Fazit}

Insgesamt zeigt sich ein umfassendes, differenziertes und durchaus heterogenes Bild von Aufgaben und Schnittstellen im Schulmanagement. Mit Blick auf die hohe Kontextabhängigkeit schulischen Leitungshandelns (vgl. Bonsen, 2016) und in der Perspektive der Educational Governance ist dieses wie folgt zu erklären: Kantonale Rahmenbedingungen (vgl. 2.1, 2.3) treffen als Steuerungsimpulse auf unterschiedliche politische, finanzielle und räumliche Voraussetzungen sowie auf personale Strukturen und teilweise individuell ausgestaltete Schnittstellen an der Einzelschule (Akteurskonstellation). Die hier vorgenommene multiperspektivische Arbeitsplatzanalyse kann zur Erforschung der so konstituierten Handlungskoordination und -logiken anhand subjektiver Perspektiven zentraler Akteure beitragen. Ein hervorzuhebendes Beispiel einer folgenreichen Struktur dieser Handlungskoordination stellen die Leitungsmodelle an der schulleitungs- und schulverwaltungsinternen Schnittstelle (Thesen 2, 3, 4) dar. Aber auch die Schnittstelle zwischen der Schulleitung und Schulverwaltung erscheint mit ungenutztem Delegationspotenzial und lokal unterschiedlichen Sekretariatspensen (These 5) 
Optimierungspotenzial zu bieten. An der Schnittstelle zwischen der Schulleitung und der Schulpflege lässt sich aus beiden Perspektiven eine unzureichende Rollenklarheit infolge kantonaler Vorgaben konstatieren, die auf der Mesoebene zu divergierenden Interpretationen und Handlungslogiken führt (Thesen 6, 7). Empirisch und die verschiedenen Akteursperspektiven bündelnd zeigt sich somit in dieser Studie: Welcher Akteur welche Aufgaben übernimmt, ist stark abhängig von der Handlungskoordination an der jeweiligen Schnittstelle im hier untersuchten Feld (These 8).

So erscheint auch plausibel, dass Belastungen verstärkt in den Schnittstellen oder bei unzureichenden Rahmenbedingungen entstehen und weniger aus den Aufgaben selbst (These 9). Aus der Perspektive zentraler Akteure ergibt sich angesichts der zwischen Makro- und Mesoebene koordinierten Anforderungen, Rahmenbedingungen und Aufgaben eine Handlungslogik, die zur Unterberücksichtigung von Aufgaben im Bereich der Schul- bzw. Qualitätsentwicklung führt (These 1). Dies ist als Resultat einer Arbeitsplatzanalyse aus Schulmanagement-Perspektive mit den gebotenen Einschränkungen (s. u.) als problematisch zu bewerten.

Die Erhebungs- und Analysemethodik kritisch in den Blick nehmend, sind die folgenden Aspekte zu erwähnen: Grundsätzlich sind trotz freiwilliger Teilnahme mögliche Antworttendenzen aufgrund sozial erwünschten Verhaltens oder des bildungspolitischen Kontexts der Erhebung nicht auszuschliessen. Auch hinsichtlich des Erhebungszeitpunktes (Mai/Juni 2016) könnte eine Verzerrung bestehen, denn zum Ende des Schuljahres äussern die Akteure einen erhöhten Aufgabenumfang im Handlungsfeld Organisation/Verwaltung. Im Kontext der Arbeitsplatzanalyse wird geraten, von substanziellen Bewertungen «allein auf der Basis von subjektiven Befragungsdaten» (Schaper, 2019, S. 395) abzusehen. Interessant und wichtig bleibt es daher, die aus diesem Grund hier als Thesen formulierten Befunde durch die Erweiterung des Forschungszugriffs einer kritischen Überprüfung zu unterziehen. Zusätzlich erscheint es relevant, entsprechende Forschungsvorhaben auf andere Kantone oder exemplarische Bundesländer in Österreich und Deutschland auszudehnen, um übergeordnete Systemabhängigkeiten zu identifizieren. Hier ist beispielsweise der Einfluss der Schulpflege zu nennen, ein zentraler Akteur im Schulmanagement im untersuchten Feld, der in anderen deutschsprachigen Ländern in dieser Form nicht vorhanden ist.

\section{Literaturverzeichnis}

Altrichter, H., \& Maag Merki, K. (2016). Steuerung der Entwicklung des Schulwesens. In H. Altrichter \& K. Maag Merki (Hrsg.), Handbuch Neue Steuerung im Schulsystem (2. Aufl., S. 1-27). VS Verl. f. Sozialwiss. https://doi:10.1007/978-3-531-18942-0 Berthel, J., \& Becker, F. G. (2013). Personal-Management (10. Aufl.). Schäffer-Poeschel.

BKS Aargau (2006). Geleitete Schule. Einrichten einer Schulleitung: Informationen zum Schulsekretariat. Departement Bildung, Kultur und Sport, Aargau, Aarau. https://www.schulen-aargau.ch/kanton/Dokumente_offen/einrichten\%20schulsekretariat. pdf [Stand: 17.09.2016].

BKS Aargau (2016). Schulführung. https://www.ag.ch/de/bks/kindergarten_volksschule/schulstruktur_organisation/schulfuehrung/schulfuehrung.jsp [Stand: 17.09.2016].

Böhm-Kasper, O. (2004). Schulische Belastung und Beanspruchung. Eine Untersuchung von Schülern und Lehrern am Gymnasium. Waxmann.

Bonsen, M. (2016). Schulleitung und Führung in der Schule. In H. Altrichter \& K. Maag Merki (Hrsg.), Handbuch Neue Steuerung im Schulsystem (2. Aufl., S. 301-323). VS Verl. f. Sozialwiss. https://doi:10.1007/978-3-531-18942-0

Bonsen, M., Iglhaut, C., \& Pfeiffer, H. (1999). Schulleitungshandeln aus Schulleitungssicht. IFS-Verlag.

Dadaczynski, K., \& Paulus, P. (2015). Healthy Principals - Healthy Schools? A Neglected Perspective to School Health Promotion. In V. Simovska \& P.M. McNamara (Hrsg.), Schools for Health and Sustainability (S. 253-273). Springer Netherlands. https:// doi:10.1007/978-94-017-9171-7_12

Dedering, K. (2012). Schulentwicklung und Schulentwicklungstheorie. Steuerung und Schulentwicklung (S. 3-46). VS Verl. f. Sozialwiss. https://doi:10.1007/978-3-531-19534-6_2

Dick, R. van, \& Stegmann, S. (2013). Belastung, Beanspruchung und Stress im Lehrerberuf - Theorien und Modelle. In M. Rothland (Hrsg.), Belastung und Beanspruchung im Lehrerberuf: Modelle, Befunde, Interventionen (S. 41-59). VS Verl. f. Sozialwiss. https://doi:10.1007/978-3-531-18990-1_3

Dlabac, O. (2016). Lokale Autonomie und Milizprinzip unter veränderten Vorzeichen: Neue Modelle der Gemeinde- und Schulorganisation. In J. Hangartner \& M. Heinzer (Hrsg.), Gemeinden in der Schul-Governance der Schweiz: Steuerungskultur im Umbruch (S. 125-145). VS Verl. f. Sozialwiss. https://doi:10.1007/978-3-658-13092-3_6

Dormann, T., Dormann, C., Zapf, D., \& Irmer, C. (2004). Belastungen im Schulsekretariat. Aufgaben und Probleme an einer pädagogischen Schnittstelle. Die Deutsche Schule, 96(3), 345-358.

Dorsemagen, C., Baeriswyl, S., \& Krause, A. (2013). Schulleitung und Gesundheit an Volksschulen des Kantons Aargau. Eine Expertise. Projektbericht für die Departemente Bildung, Kultur und Sport (BKS) sowie Gesundheit und Soziales (DGS) des Kantons Aargau. https://irf.fhnw.ch/handle/11654/3187 [Stand: 17.08.2016].

Flick, U. (2014). Qualitative Sozialforschung. Eine Einführung. (6. Aufl.). Rowohlt. 
Greif, S. (2021). Arbeitsanalyse. Dorsch Lexikon der Psychologie. Hogrefe. https://dorsch.hogrefe.com/stichwort/arbeitsanalyse [Stand: 21.04.2021].

Greubel, M. (2017). Die Einführung einer erweiterten Schulleitung an eigenverantwortlichen beruflichen Schulen. FAU Nürnberg.

Heinzer, M. (2016). Gestaltungsspielräume der Gemeinden - Schulsteuerung zwischen Subsidiarität und Hierarchie. In J. Hangartner \& M. Heinzer (Hrsg.), Gemeinden in der Schul-Governance der Schweiz: Steuerungskultur im Umbruch (S. 101-123). VS Verl. f. Sozialwiss. https://doi:10.1007/978-3-658-13092-3_5

Huber, S. G. (2011a). School Governance in Switzerland: Tensions between New Roles and Old Traditions. Educational Management Administration \& Leadership, 39(4), 469-485.

Huber, S. G. (2011b). Steuergruppen - eine Einführung. Handbuch für Steuergruppen. Grundlagen für die Arbeit in zentralen Handlungsfeldern des Schulmanagements (S. 1-10). Wolters Kluwer.

Huber, S. G. (2013). Forschung zu Belastung und Beanspruchung von Schulleitung. In S.G. Huber (Hrsg.), Jahrbuch Schulleitung 2013 (S. 222-240). Wolters Kluwer.

Huber, S. G., Hader-Popp, S., \& Schneider, N. (2014). Qualität und Entwicklung von Schule: Basiswissen Schulmanagement. Beltz. Jahn, R. (2017). Im Sog des Infantilen: Schulleitung als Beruf. VS Verl. f. Sozialwiss. https://doi:10.1007/978-3-658-14848-5

Krause, A., Baeriswyl, S., \& Dorsemagen, C. (2013). Die Arbeits- und Gesundheitssituation von Schulleitungen. Befunde und Thesen zu situativen und personalen Ressourcen. In S.G. Huber (Hrsg.), Jahrbuch Schulleitung 2013 (S. 241-258). Wolters Kluwer.

Landert, C. (2009). Arbeitssituation der Schulleitungen im Kanton Thurgau. Evaluation. Amt für Volksschule und Kindergarten.

Mayring, P. (2015). Qualitative Inhaltsanalyse: Grundlagen und Techniken. (12. Aufl.). Beltz.

Pekruhl, U., Dorsemagen, C., Kölliker, A., \& Krause, A. (2011). Arbeitszeit und Arbeitsorganisation der Schulleitungen an den Volksschulen des Kantons Basel-Landschaft. Projektbericht für die Bildungs-, Kultur-und Sportdirektion Basel-Landschaft. FHNW.

Quesel, C., Näpfli, J., Kübler, D., Buser, P., \& Gundelach, B. (2014). Schulkommissionen und Elterngremien im Urteil professioneller Schulleitungen. PH FHNW/Zentrum für Demokratie Aarau. https://doi:10.26041/fhnw-186

Ramseier, E., \& Brühwiler, C. (2003). Herkunft, Leistung und Bildungschancen im gegliederten Bildungssystem. Vertiefte PISAAnalyse unter Einbezug der kognitiven Grundfähigkeiten. Schweizerische Zeitschrift für Bildungswissenschaften, 25(1), 23-58. https://doi.org/10.24452/sjer.25.1.4646

Schaper, N. (2019). Arbeitsanalyse und -bewertung. In F.W. Nerdinger, G. Blickle \& N. Schaper (Hrsg.), Arbeits- und Organisationspsychologie (S. 385-410). Springer. https://doi:10.1007/978-3-662-56666-4_21

Trachsler, E., Brüggen, S., Ulich, E., Nido, M., Wülser, M., \& Voser, S. (2007). Arbeitsbedingungen, Belastungen und Ressourcen in der Thurgauer Volksschule. Teilstudie Schulbehörden. Ergebnisse der zweiten Erhebung. PH Thurgau. http://edudoc.ch/getfile. py? recid $=26649$ [Stand: 02.12.2020].

Trachsler, E., Ulich, E., Inversini, S., Wülser, M., \& Dangel, M. (2005). Arbeitsbedingungen, Belastungen und Ressourcen in der Thurgauer Volksschule. Teilstudie Schulbehörden. Ergebnisse der Analyse der ersten Teilstudie. PH Thurgau.

Tulowitzki, P., \& Pietsch, M. (2020). Stichwort: Lernzentriertes Leitungshandeln an Schulen - Leadership for Learning. Zeitschrift für Erziehungswissenschaft, 23(5), 873-902. https://doi:10.1007/s11618-020-00964-8

Warwas, J. (2012). Berufliches Selbstverständnis, Beanspruchung und Bewältigung in der Schulleitung. VS Verl. f. Sozialwiss. https:// doi:10.1007/978-3-531-19300-7

Wissinger, J. (2014). Schulleitung und Schulleitungshandeln. In E. Terhart, H. Bennewitz \& M. Rothland (Hrsg.), Handbuch der Forschung zum Lehrerberuf (2. Aufl., S. 144-176). Waxmann.

Schlagworte: Schulmanagement; Schnittstellen; Schulleitung; Schulsekretariat; Schulpflege

\section{La gestion scolaire entre la gestion de la vie quotidienne et le développement stratégique de l'école - thèses d'une analyse qualitative multi-perspective du lieu de travail}

Résumé

Cet article utilise une étude par entretiens pour répondre aux questions de savoir comment les tâches et les interfaces dans la gestion des écoles sont structurées. Les résultats de 18 entretiens d'experts avec la direction de l'école ainsi que le personnel de secrétariat et les membres de l'administration de l'école sont présentés et discutés. Il s'ensuit qu'une forte intensité de la charge de travail ainsi qu'un manque de temps permanent conduisent inévitablement à une priorisation de la garantie de la routine scolaire quotidienne, où les tâches pertinentes pour la qualité de l'école sont systématiquement négligées. Compte tenu des ambiguïtés et du potentiel d'optimisation de la division du travail, il est avancé que les interfaces représentent un facteur d'influence décisif pour l'action dans la gestion des écoles.

Mots-clés: Gestion des écoles ; interfaces ; direction d'un établissement scolaire ; secrétariat des écoles ; administration des écoles 


\section{La direzione scolastica tra gestione dei compiti quotidiani e sviluppo strategico della scuola - tesi di un'analisi qualitativa multi-perspettiva del posto di lavoro}

\section{Riassunto}

Attraverso uno studio di interviste questo articolo indaga come sono strutturati i compiti e le interfacce nella gestione della scuola. A tal fine, sono presentati e discussi i risultati di 18 interviste guidate con la direzione della scuola, con il personale di segreteria e con i membri dell'amministrazione scolastica. Lo studio mostra come un'alta intensità del carico di lavoro e una permanente mancanza di tempo conducano inevitabilmente ad attribuire priorità alla routine scolastica quotidiana, e, di conseguenza, a trascurare sistematicamente i compiti rilevanti per la qualità della scuola. In considerazione delle ambiguità e del potenziale d'ottimizzazione tramite la divisione del lavoro, si sostiene che le interfacce rappresentino un fattore d'influenza decisivo per l'azione nella gestione della scuola.

Parole chiave: Gestione scolastica; interfacce; direzione; segreteria scolastica; amministrazione scolastica

\section{School leadership in between daily tasks and strategic school improvement - theses from a qualitative workplace analysis from the perspective of key actors}

Summary

With a multi-perspective approach, we explore tasks and interfaces in school management regarding their general arrangement and functionality. Results of 18 structured interviews with school leaders, secretary staff and members of school boards are presented and discussed. It can be shown that stress, workload and time pressure convey dysfunctional effects: Hence, the key actors prioritise continuity and provision of essential functioning of their schools. Therefore, tasks concerning quality management and school improvement as well as personnel management become systematically neglected. Furthermore, we identify various role-related ambiguities and potentials for improving task distribution. When reflecting on the derived theses, it is argued that interfaces constitute a vital impact on school management.

Keywords: School management; interfaces; school leadership; school office; school board

Christoph Kruse, M. Ed., Studium der Erziehungswissenschaft, Sozialwissenschaften und Mathematik in Magdeburg, Bielefeld und London; wissenschaftlicher Mitarbeiter in der Arbeitsgruppe Allgemeine Didaktik und Unterrichtsforschung am Institut für Erziehungswissenschaft der Westfälischen Wilhelms-Universität Münster. Forschungsgebiete: Schulleitungs- und Schulentwicklungsforschung sowie Forschung zum Lehrer/innenberuf und zur Lehrer/-innenbildung.

Institut für Erziehungswissenschaft, WWU Münster, Bispinghof 5/6, D-48143 Münster

E-Mail: christoph.kruse@uni-muenster.de

Stephan Gerhard Huber, Prof. Dr., Leiter des Instituts für Bildungsmanagement und Bildungsökonomie IBB der Pädagogischen Hochschule Zug. Arbeitsschwerpunkte: Systemberatung, Bildungsmanagement, Bildungsqualität, Educational Change, Professionalisierung von pädagogischem (Führungs-)Personal, internationalvergleichende Bildungsforschung sowie Jugendforschung.

PH Zug, Zugerbergstrasse 3, CH-6300 Zug

E-Mail: stephan.huber@phzg.ch 